\title{
11 ADHS im forensisch-psychiatrischen Kontext
}

\author{
Jana Engel, Martin Neumeyer, Elmar Habermeyer und Detlef Schläfke
}

Die ADHS ist mit einer Prävalenz von 6-10\% die häufigste kinderpsychiatrische Erkrankung. Bei ein bis zwei Dritteln der Betroffenen kommt es zu einer Persistenz der Symptome ins Erwachsenenalter, die Prävalenz wird auf 2-6\% geschätzt. Innerhalb forensischer Populationen zeigen sich in Deutschland Prävalenzen von bis zu $45 \%$.

In der vorliegenden Untersuchung wurden bei $65,7 \%$ der Insassen einer forensischen Klinik mittels Wender-Utah-Rating-Scale (WURS) und ADHS-SB Symptome aus dem Spektrum einer ADHS gefunden. Anschließend wurde diese Gruppe $(n=34)$ mit nicht-delinquenten $(n=31)$ Patienten verglichen, welche sich bereits aufgrund einer vorausgehend diagnostizierten ADHS in ambulanter Behandlung befanden.

Es zeigten sich signifikante Unterschiede in der Entwicklung und im Vorhandensein psychiatrischer Komorbiditäten. Die kindliche Entwicklung der delinquenten Patienten war signifikant häufiger von Vernachlässigungen geprägt. Außerdem zeigten sich signifikant häufiger komorbide Verhaltensprobleme oder dissoziale Persönlichkeitsstörungen innerhalb der forensischen Gruppe. Bei der Intelligenzprüfung nach HAWIE fanden sich signifikant höhere Werte bei den nicht-delinquenten Patienten.

Die Untersuchungsergebnisse zeigen, dass nicht das Vorliegen einer ADHS ausschlaggebend für eine spätere Delinquenz ist, sondern dass die Ursache eher in komorbiden Verhaltensstörungen und dissozialen Persönlichkeitsauffälligkeiten sowie einer gestörten Entwicklung zu finden ist. In diesem Rahmen empfiehlt es sich, Patienten mit ADHS besonders auf komorbide Störungen zu untersuchen und Kindern aus Problemfamilien eine besondere Unterstützung zukommen zu lassen. 
Die Aufmerksamkeitsdefizit-/Hyperaktivitätsstörung (ADHS) ist in Deutschland und auch weltweit die häufigste kinder- und jugendpsychiatrische Erkrankung. Ihre Prävalenz wird auf 6-10\% geschätzt (Wender 2000). Lange Zeit ging man vor allem in Deutschland davon aus, diese Krankheit beträfe nur Kinder und Jugendliche. In Amerika wies vor allem Paul H. Wender schon in den 8oer-Jahren darauf hin, dass diese Störung persistieren und auch im Erwachsenenalter gravierende psychosoziale Auswirkungen haben kann. Es sollen ein bis zwei Drittel der betroffenen Kinder auch als Erwachsene noch erhebliche Symptome aufweisen. Die Prävalenz einer ADHS im Erwachsenenalter wird auf 2-6\% geschätzt (Krause und Krause 2003). Allgemein wird eine deutliche Bevorzugung des männlichen Geschlechts (3:1 bis 4:1) angegeben. Wender führt dies darauf zurück, dass in Studien zu wenige weibliche Patienten erfasst wurden, da bei diesen die Unaufmerksamkeit und weniger die Hyperaktivität/Impulsivität im Vordergrund steht (Krause und Krause 2003).

Die ICD-1o ermöglicht folgende diagnostische Eingruppierungsmuster:

- F go hyperkinetische Störungen: mit in Bezug auf Alter und Entwicklungsstand

- beeinträchtigter Aufmerksamkeit und Hyperaktivität (F go.o einfache Störung),

- kombiniert mit einer Störung des Sozialverhaltens (F 90.1).

Das DSM-IV (American Psychiatric Association 1994) differenziert im Gegensatz zur ICD-10 drei Untergruppen:

- Typ 1: Mischtypus, es liegen jeweils mindestens sechs von neun Symptomen der Hyperaktivität/Impulsivität sowie der Aufmerksamkeitsstörung vor,

- Typ 2: vorwiegend unaufmerksamer Typus, es liegen mindestens sechs Symptome der Aufmerksamkeitsstörung vor, jedoch weniger als sechs Symptome der Hyperaktivität/Impulsivität,

- Typ 3: vorwiegend hyperaktiv-impulsiver Typus, es treffen mindestens sechs Kriterien der Hyperaktivität/Impulsivität zu, jedoch weniger als sechs der Aufmerksamkeitsstörung.

\subsection{Zur ADHS-Entwicklung und -Symptomatik}

Die ADHS ist eine Erkrankung mit starker genetischer Verankerung. So belegten Zwillingsstudien (Todd et al. 2001), Familienuntersuchungen (Faraone et al. 2000) und Adoptivstudien (Cadoret und Stewart 1991) eine erhebliche hereditäre Komponente. Konkordanzraten bei monozygoten Zwillingen werden mit 6o-80\% angegeben (Smidt et al. 2003). Das genetische Zusammenspiel ist nach neuesten Untersuchungen sehr komplex, es wird eine Interaktion multipler Gene vermutet (Faraone et al. 2005). Als Kandidatengene, welche die Wahrscheinlichkeit eines Auftretens einer ADHS zu erhöhen scheinen, 
gelten das Dopamintransporter-(DAT1-)Gen auf Chromosom 5, das DopaminD4-Rezeptor-(DRD4-)Gen auf Chromosom 11 und das D2-Dopamin-RezeptorGen (Faraone und Biederman 1998).

Auf molekularer Ebene werden Funktionsabweichungen verschiedener Transmittersysteme angenommen, vor allem im Bereich der biogenen Amine Dopamin und Noradrenalin wird eine Störung vermutet (Faraone und Biederman 1998), seit neuester Zeit auch im Serotoninhaushalt (Rösler 2001).

In strukturell-bildgebenden Verfahren und mittels SPECT zeigten sich hirnorganische Auffälligkeiten, v. a. Volumenminderungen im rechten präfrontalen Kortex, des Globus pallidum, des Striatums (v. a. Nucleus caudatus) und in Kleinhirnregionen. Außerdem fand sich eine erhöhte Dichte von Dopamintransportern im Striatum sowie eine verminderte Durchblutung im Frontallappenbereich und im Striatum, vor allem rechts, welche sich nach Methylphenidatgabe normalisierte (Rubia et al. 1999). In einer PET-Studie wiesen Zametkin et al. (1993) einen verminderten Clucosemetabolismus im gesamten frontalen Kortex nach, mit einer Betonung des linken prämotorischen und des superioren präfrontalen Kortex. Den gesamten Metabolismus betreffend zeigten sich jedoch keine signifikanten Unterschiede.

Die Symptomatik unterliegt während des Heranwachsens Modifikationen, das Bild des hyperaktiven, herumzappelnden Kindes ist beim Erwachsenen kaum anzutreffen. Jedoch sind die wesentlichen Symptome wie Aufmerksamkeitsstörung, motorische Störung, Impulsivität, Desorganisation, Probleme im sozialen Umfeld, Schwierigkeiten in persönlichen Beziehungen, emotionale Störungen und Stressintoleranz auch bei den betroffenen Erwachsenen zu beobachten. Zurzeit sind die von Wender entwickelten Utah-Kriterien am besten geeignet, die zugrunde liegenden Symptomgruppen aufzuzeigen (Übersicht in Wender 2000). Die Symptomatik der ADHS bei Erwachsenen äußert sich wie folgt:

\section{Aufmerksamkeitsstörung}

- Unfähigkeit, sich auf Gespräche zu konzentrieren

- erhöhte Ablenkbarkeit

- Schwierigkeiten, sich auf schriftliche Dinge oder Aufgaben zu konzentrieren

- Vergesslichkeit

- häufiges Verlegen von Gegenständen

\section{Motorische Störungen}

- Gefühl der inneren Unruhe

- Unfähigkeit, sich zu entspannen, Nervosität

- Unfähigkeit, längere Zeit sitzende Tätigkeiten durchzuhalten

- dysphorische Stimmungslagen bei Inaktivität 


\section{Affektlabilität}

- abrupter Wechsel zwischen normaler Stimmung und Niedergeschlagenheit sowie leichtgradigen Erregungserscheinungen

- Niedergeschlagenheit wird vom Patienten oft als Zustand der Unzufriedenheit, Lustlosigkeit und Langeweile beschrieben

- Stimmungswechsel dauern einige Stunden bis maximal einige Tage

- im Gegensatz zur Depression kein ausgeprägter Interessenverlust oder somatische Begleiterscheinungen

- Stimmungswechsel sind auf auslösende Ereignisse zurückzuverfolgen, z. T. treten sie auch spontan auf

\section{Desorganisation}

- Aktivitäten werden unzureichend geplant und organisiert, v. a. in der Schule, bei der Arbeit oder in der Haushaltsführung

- begonnene Arbeiten werden nicht zu Ende gebracht und ständig neue Aufgaben in Angriff genommen, es kommt zu Unterbrechungen und Verzögerungen

- unsystematische Problemlösungsstrategien

- Schwierigkeiten in der zeitlichen Organisation, Unfähigkeit, Termine oder Zeitpläne einzuhalten

\section{Affektkontrolle}

- andauernde Reizbarkeit, auch aus geringem Anlass, verminderte Frustrationstoleranz und Wutausbrüche

- typisch ist eine erhöhte Reizbarkeit im Straßenverkehr im Umgang mit anderen Verkehrsteilnehmern

- nachteilige Auswirkungen der mangelnden Affektkontrolle auf Beziehungen

Impulsivität

- Dazwischenreden, Unterbrechen anderer Gespräche und Ungeduld, impulsive Geldausgaben

- schnell gefasste Entschlüsse

- Unfähigkeit, Handlungen im Verlauf zu protrahieren

Emotionale Überreagibilität

- Unfähigkeit, mit alltäglichen Stressoren umzugehen, der Patient reagiert inadäquat und überschießend niedergeschlagen, verwirrt, unsicher, ärgerlich oder ängstlich

- Patienten beschreiben sich als schnell „belästigt“ oder „gestresst“ 
Die Diagnose der ADHS im Erwachsenenalter ist eine klinische Diagnose, sie erfolgt mit Hilfe des Interviews mit dem Patienten, den darin enthaltenen Angaben zur Eigen- und Familienanamnese, den psychopathologischen Befunden sowie der erfassbaren Symptome und deren Verlauf. Zusätzlich sollten Komorbiditäten beschrieben werden. Hilfreich ist es, wenn Fremdbeobachtungen vorliegen. Die Diagnose kann mittels diverser Fragebögen unterstützt werden. Die Diagnosestellung einer ADHS im Erwachsenenalter erfordert, dass sowohl aktuell die Kriterien einer ADHS durchgehend erfüllt sind als auch in Kindheit und Jugend waren (Krause 2006).

Zur retrospektiven Erfassung kindlicher Symptome eignet sich die WenderUtah-Rating-Scale (WURS), deren deutsche Version von Groß et al. (1999) als geeignetes Instrument bestätigt wurde. Diese wurde von Retz-Junginger et al. (2002) zu einer verkürzten Version zusammengefasst (WURS-k). Zur Einschätzung der aktuellen Symptomatik sind die für deutschsprachige Länder entwickelten ADHS-SB und ADHS-DC geeignet, die sich an den Kriterien der ICD-10 und DSM-IV orientieren (Rösler et al. 2004; Rösler et al. 2006).

Auch andere ADHS-Ratinginstrumente, wie die von Connors et al. (1999) entwickelte „Conners’ Adult ADHD Rating Scale“ (CAARS), welche auch als Fremdbeurteilungsbogen vorliegt, die von Brown entwickelte „Brown ADD Scale“ (BADDS) oder das „Wender-Reimherr-Interview“ (WRI) werden zur Erwachsenendiagnostik genutzt.

Zur differentialdiagnostischen Abklärung sind eine zusätzliche orientierende Intelligenz- und Gedächtnisdiagnostik (z. B. HAWIE, WMS-R) sowie neuropsychologische Tests (z. B. Wisconsin Card Sorting Test, Continuous Performance Test, D2-Test, Trail Making Test, Attention Network Test) zu empfehlen. Die neuropsychologischen Testergebnisse sind jedoch nicht obligatorisch für die Diagnosestellung.

Die Behandlung einer ADHS muss nicht notwendigerweise medikamentös erfolgen. Je nach Ausprägung der Symptome und Einschränkungen in verschiedenen Lebensbereichen kann sie rein psychotherapeutisch (bei leichter Ausprägung) oder pharmakotherapeutisch (bei schwerer Ausprägung) oder kombiniert erfolgen.

Bei medikamentöser Therapie ist die Gabe von Stimulanzien (Methylphenidat) Mittel der ersten Wahl (Faraone et al. 2004). Methylphenidat stimuliert die Ausschüttung von Dopamin und Noradrenalin aus den präsynaptischen Vesikeln und hemmt die Wiederaufnahme. Die Dosierung gestaltet sich im Erwachsenenalter schwieriger als im Kindesalter, da die Verstoffwechselung größeren Einflüssen unterliegt Retardiertes Methylphenidat, welches Reboundphänomene während des Tagesverlaufs verhindert, ist für die Therapie Erwachsener in Deutschland noch nicht zugelassen, im Kindesalter findet es bereits Verwendung (Sobanski und Alm 2005). Bei Nichtansprechen auf die Therapie kann eine Gabe von D-L-Amphetamin zur Besserung der Symptome führen.

Nach neuesten Erkenntnissen führt auch der Noradrenalinwiederaufnahmehemmer Atomoxetin zu positiven Ergebnissen, vor allem bei Vorliegen einer komorbiden Suchterkrankung, ebenso Serotoninwiederaufnahmeinhibitoren, 
welche zusätzlich die Komorbidität einer depressiven Erkrankung bessern (Spencer und Biederman 2000).

Ein häufig unterstelltes erhöhtes Potenzial bei der Behandlung mit Medikamenten, später eine Sucht zu entwickeln, besteht nicht (Lojewski et al. 2002). Auch eine Abhängigkeit durch Methylphenidat selbst konnte nicht nachgewiesen werden. Es kommt weder zur Toleranzentwicklung noch zum Auftreten von Entzugssymptomen nach Absetzen des Medikamentes. Ein protektiver Effekt hinsichtlich der Stimulanzienbehandlung bei Kindern mit ADHS, bezogen auf eine spätere Entwicklung von Suchterkrankungen, scheint nach aktueller Studienlage vorhanden zu sein (Davids und Gastpar 2003), auch zusätzliche Störungen des Sozialverhaltens werden positiv beeinflusst (Retz et al. 2007).

\subsection{Funktionelle Auswirkungen, Komorbidität und Kontext zur forensischen Psychiatrie}

Eine Relevanz der ADHS zeigen Untersuchungen, die verdeutlichen, dass die Krankheit zu einer erheblichen Einschränkung in Bezug auf die Entwicklung eines Menschen in den unterschiedlichsten Lebensbereichen führt. Es kommt zu häufigeren Schulverweisen, Suspendierungen vom Unterricht, Schul- und Studienabbrüchen, Kündigungen oder freiwilligen Berufswechseln; das Risiko für Unfälle, besonders im Verkehr, ist erhöht, ebenso wie die Scheidungsanamnese (Murphy und Barkley 1996). Die Cambridge-Studie zur Delinquenzentwicklung bestätigte, dass das Syndrom Impulsivität-Hyperaktivität-Konzentrationsstörungen im Kindesalter, mit schon früh einsetzenden Störungen in der sozialen Anpassung, ein valider Prädiktor für spätere Aggressivität und Gewalttätigkeit ist (Farrington 2000).

Des Weiteren leiden bis zu 77 \% der erwachsenen Patienten an komorbiden psychiatrischen Erkrankungen (Biederman et al. 1993). Vor allem Substanzmissbrauch (30-50\%), affektive Störungen (20-30\%), Angsterkrankungen (20$30 \%)$, Verhaltensstörungen (30-50\%) und Persönlichkeitsstörungen (50\%) (insbesondere antisoziale Persönlichkeitsstörung und emotional-instabile Persönlichkeitsstörung) dominieren.

Besonders im Bereich des Substanzmissbrauchs und der Entwicklung des oppositionellen Verhaltens bzw. einer antisozialen Persönlichkeitsstörung ergeben sich Bezugspunkte zur forensischen Psychiatrie.

Aber auch allgemein steht die Symptomatik der ADHS im Verdacht, ein bedeutsamer Faktor für eine spätere Delinquenzentwicklung zu sein. Eine kindliche ADHS und komorbide Persönlichkeits- und Verhaltensstörungen gelten als Prädiktoren für eine spätere Delinquenz und Risikofaktoren für eine Rezidivdelinquenz (Rösler 2001; Barkley et al. 1990; Blocher et al. 2001). Vor allem das bei der ADHS erhöhte Risiko der Entwicklung einer oppositionellen Verhaltensstörung oder einer antisozialen Persönlichkeitsstörung (Barkley et al. 1990) gewinnt aus forensischer Sicht an Bedeutung. In der Cambridge-Studie 
wurden diese Störungen als valide Prädiktoren für spätere Delinquenz belegt (Farrington 200o). Auch der mit der ADHS assoziierte Drogenmissbrauch bzw. die Drogenabhängigkeit sind von forensischer Relevanz. Studien zeigen ein bis ums Vierfache erhöhtes Vorkommen einer Drogenabhängigkeit bei vorliegender ADHS (Gittelman et al. 1985; König et al. 2007; Murphy und Barkley 1996), vor allem der Missbrauch von Cannabis steht im Vordergrund (Ohlmeier, et al. 2005). Dies wirkt sich wiederum auf eine Beschaffungskriminalität im Sinne von Raub und Eigentumsdelikten aus, die bei straffälligen ADHSPatienten zusätzlich zu Drogendelikten im Vordergrund stehen (Rösler 2001). Aber auch im Bereich der Sexualdelinquenz gibt es Hinweise darauf, dass die Diagnose einer ADHS mit Paraphilie und devianten, aggressiven Formen sexueller Impulsivität korreliert (Blocher. 2001).

In verschiedenen Studien zeigte sich, dass mit einem früheren Auftreten von Störungen des Sozialverhaltens, delinquenten Verhaltensweisen, und der Persistenz dieser Störungen zu rechnen ist, wenn eine ADHS vorliegt (Retz und Rösler 2006). Umgekehrt zeigten Untersuchungen an Gefängnispopulationen Deutschlands Prävalenzraten der ADHS von bis zu $45 \%$. Blocher et al. (2001) fanden bei einer Gruppe von Sexualstraftätern eine kindliche Prävalenz von 27,6\%, dabei kam es zu einem um zehn Jahre früheren Beginn der kriminellen Karriere, wenn Symptome der ADHS in der Kindheit vorlagen. Ziegler et al. (2003) ermittelten bei $43 \%$ der untersuchten JVA-Insassen retrospektiv ADHS-Symptome. Vor allem bei Rezidivtätern fanden sich gesteigert Symptome des Krankheitsbildes. Insbesondere bei der Kombination von Verstößen gegen das Betäubungsmittelgesetz, Eigentumsdelikten und Körperverletzungen waren vermehrt Insassen mit kindlicher ADHS auffällig. Rösler et al. (2004) wiesen eine Prävalenz der ADHS von $45 \%$ nach DSM-IV-Kriterien und $22 \%$ nach ICD-10-Kriterien bei der Untersuchung von JVA-Insassen nach. Auch im Rahmen eigener Voruntersuchungen zeigte sich eine erhöhte Prävalenz der ADHS bei Maßregelvollzugspatienten.

Als Grund für die hohe Prävalenz einer ADHS in forensischen Populationen nennen Ziegler et al. (2003), dass in Justizvollzugsanstalten eine Selektierung von Personen stattfindet, die auch als Erwachsene unter der Symptomatik leiden. Es kommt durch impulsives, unüberlegtes Verhalten, plötzliche Gefühlsausbrüche und Stimmungsschwankungen zu gehäuften Konflikten mit der Umwelt und dem Gesetz. Das Risiko für Festnahmen und Inhaftierungen scheint bei Patienten mit einer ADHS deutlich erhöht zu sein (Rasmussen und Gillberg 2000).

Auf der anderen Seite gibt es viele Patienten mit einer ADHS, die trotz starker Ausprägung der Erkrankung keine delinquente Entwicklung aufweisen und ihren Lebensweg ohne größere Probleme gehen. Ziel dieser Studie ist, Zusammenhänge zwischen Klienten mit einer ADHS und einer delinquenten bzw. nichtdelinquenten Entwicklung zu untersuchen. Daneben sollen Faktoren herausgearbeitet werden, die eine delinquente Entwicklung bei vorliegender ADHS voraussagen oder protektive Funktionen haben. 


\subsection{Methodik}

Es wurden 67 Patienten eines Entziehungsmaßregelvollzugs nach $\$ 64$ StCB auf das Vorliegen einer ADHS untersucht. Den Patienten wurden als Eingangsscreening die lange Version der Wender-Utah-Rating-Scale (WURS) und der ADHS-SB-Fragebogen vorgelegt. Beides sind Selbstbeurteilungsfragebögen, die WURS für die retrospektive Diagnostik einer kindlichen ADHS, die ADHS-SB zum Einschätzen einer aktuellen Symptomatik. Die Patienten müssen auf einer Skala von o bis 3 (ADHS-SB) bzw. o bis 4 (WURS) einschätzen, ob die genannten Symptome auf sie zutreffen bzw. zutrafen. Ein Überschreiten des Cut-Off von go Punkten in der WURS und von 15 Punkten in der ADHS-SB spricht für das Vorhandensein einer ADHS. In dieser Untersuchung wurde der Cut-Off jedoch auf 80 Punkte (WURS) und 10 Punkte (ADHS-SB) gesenkt, um ein größeres Patientenkollektiv in das Screening einzuschließen. Sämtliche Patienten, die in einem dieser beiden Testbögen den geforderten Cut-Off erreichten, wurden in die Untersuchung eingeschlossen.

Die Kontrollgruppe besteht aus 31 männlichen psychiatrischen Patienten, bei denen bereits eine ADHS diagnostiziert wurde und die sich zum Untersuchungszeitpunkt mit ihrer Erkrankung in ambulanter Behandlung in einer ADHS-Sprechstunde befanden. Da sich diese Patienten selbstständig in Therapie begeben haben, ist in dieser Gruppe mit einer stärkeren Ausprägung der ADHS-Symptomatik zu rechnen. Von diesen Patienten bearbeiteten 24 die kurze Version der Wender-Utah-Rating-Scale (WURS-k), bei der ein Erreichen von 30 Punkten für ein Vorliegen der ADHS in der Kindheit spricht, und sechs die lange Version der WURS. Der Cut-Off wurde, wie bei den Patienten der Forensik, auf 80 Punkte heruntergesetzt.

Das Herabsetzen des Cut-Off führte zu einer Vergrößerung der Stichprobe, allerdings muss der Einengung der Diagnostik hierbei Rechnung getragen werden. Es empfiehlt sich, bei den Patienten der Forensik nicht mehr von der Diagnose einer ADHS zu sprechen sondern von der Untersuchung von Symptomen aus dem Spektrum der ADHS.

Eine weiterführende Persönlichkeitsdiagnostik wurde mittels Temperament- und Charakter-Inventar (TCI) durchgeführt. Zusätzlich wurde zur Erfassung des Intelligenzquotienten bei beiden Patientengruppen der HamburgWechsler-Intelligenz-Test (HAWIE) sowie der Mehrfachwahl-Wortschatz-Intelligenztest (MWT-B) angewendet.

Die statistische Berechnung erfolgte mittels SPSS 15.o. Die Chi-QuadratBerechnungen zum Vergleich der beiden Gruppen wurden mit dem exakten Test nach Fischer durchgeführt. Bei größeren Kreuztabellen erfolgte die Auswertung mittels Pearsons Chi-Quadrat. Zum Teil wurde nur die Nullhypothese der Gleichheit der Mittelwerte geprüft. Konnte Normalverteilung angenommen werden (die Prüfung erfolgte mittels Chi-Quadrat-Test), so wurde der t-Test angewandt, andernfalls der Mann-Whitney-U-Test. Multiple Mittelwertvergleiche wurden mittels einfaktorieller univariater Varianzanalyse durchgeführt. 
Die Prüfung des Vorhandenseins einer Korrelation, d. h. der Ablehnung der Nullhypothese, dass der Korrelationskoeffizient gleich o ist, wurde je nach Art der Merkmale nach Pearson (bei Normalverteilung) bzw. Kendall's tau_b (keine Normalverteilung) durchgeführt.

Eine Überprüfung signifikanter Einflüsse mehrerer Faktoren bzw. von diversen Faktorenkombinationen wurde mit mehrfaktorieller univariater Varianzanalyse durchgeführt.

\subsection{Ergebnisse}

Im Rahmen einer Stichtagserhebung in der Forensischen Klinik konnten ausgehend von 67 forensischen Patienten bei insgesamt 44 (65,7\%) mittels der WURS oder der ADHS-SB Symptome einer Aufmerksamkeitsdefizit-/Hyperaktivitätsstörung festgestellt werden. Nach dem Ausscheiden von zehn dieser Patienten im Laufe der Untersuchungen bestand das endgültige Kollektiv aus 34 Patienten im Alter von 17 bis 52 Jahren, darunter befanden sich zwei Frauen (Altersdurchschnitt: 28 Jahre).

Von diesen 34 Patienten erreichten 21 (61,8\%) einen Cut-Off von 8o Punkten in der WURS, was für das Vorliegen einer ADHS-Symptomatik in der Kindheit spricht. 17 dieser forensischen Insassen (50\%) erzielten den Cut-Off von zehn Punkten in der ADHS-SB, was ein Fortbestehen der Symptome bis ins Erwachsenenalter anzeigt. 13 Patienten $(38,2 \%)$ zeigten nur in der ADHS-SB positive Ergebnisse, nicht jedoch in der WURS. Bei vier Patienten ließen sich die Symptome der ADHS nur in der Kindheit nachweisen, sie erreichten den Cut-Off in der ADHS-SB nicht.

Insgesamt erzielten 30 Patienten $(88,2 \%)$ den Cut-Off in der ADHS-SB. Von diesen zeigten fünf kein vollständiges Bild der ADHS, sondern wiesen nur in einzelnen Merkmalsbereichen hohe Punktwerte auf.

Die Vergleichsgruppe der psychiatrischen Patienten bestand aus 31 männlichen Patienten im Alter von 19 bis 48 Jahren (Altersdurchschnitt: 29 Jahre) aus der ADHS-Sprechstunde der psychiatrischen Klinik

Bei 25 dieser Patienten wurde eine kindliche ADHS festgestellt, sie erfüllten entweder den geforderten Cut-Off von 30 Punkten in der WURS-k oder den Cut-Off von 80 in der WURS. Der Cut-Off von 10 Punkten in der ADHS-SB wurde von allen Patienten erreicht.

Bei der Auswertung der Ergebnisse des TCI (s. Abb. 20) zeigten sich keine signifikanten Unterschiede im Hinblick auf die Prozentwerte in den einzelnen Dimensionen (Mann-Whitney-U-Test). Im Neugierverhalten erreichten die Patienten der Forensik im Durchschnitt 64\%, die Patienten der Psychiatrie $76 \%$. Es findet sich allerdings in der Cruppe der forensischen Patienten ein deutliches Überwiegen von explosiven Temperamentstrukturen.

In der Bearbeitung des MWT-B erzielten die Patienten der Forensik einen durchschnittlichen IQ von 97,4; die Patienten der Psychiatrie erlangten einen durchschnittlichen IQ von 104,2. Es ergeben sich im Vergleich tendenzielle 
Unterschiede. In der Auswertung des HAWIE zeigten sich signifikante Unterschiede, die forensischen Patienten schnitten hierbei deutlich schlechter ab (Mittelwert: 88 vs. 116,8; t-Test). Die einzelnen Werte beschreibt die Abbildung 21.

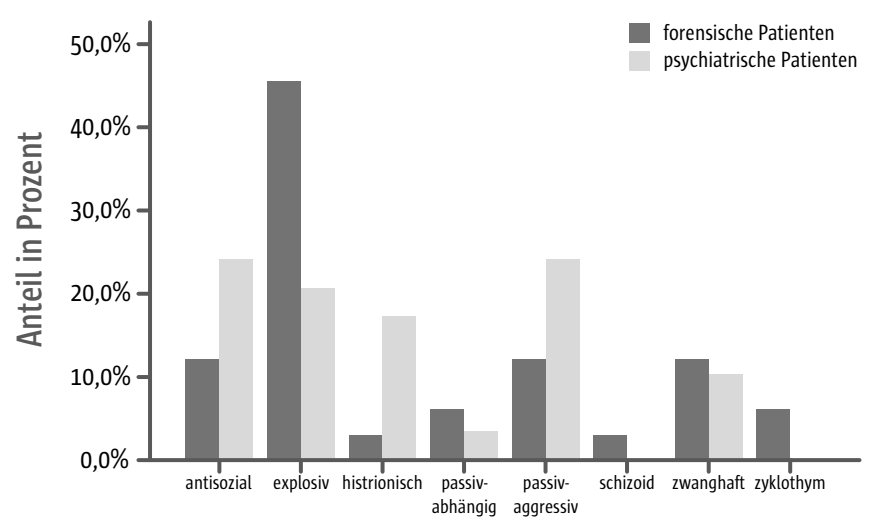

Abb. 20 Temperamentstruktur nach TCl
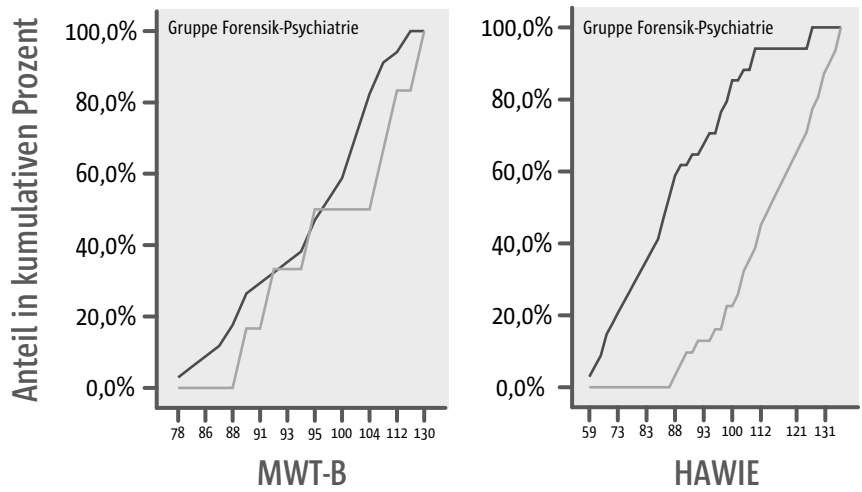

Abb. 21 Intelligenzquotient nach MWT-B/HAWIE

Bei Betrachtung des familiären Hintergrunds ergeben sich folgende Daten für beide Gruppen.

In der Schwangerschaftsanamnese existieren keine signifikanten Unterschiede bezüglich pathologischer Ereignisse $15,4 \%$ in der forensischen Patientengruppe vs. $14,8 \%$ in der psychiatrischen Patientengruppe).

Hinsichtlich der Geburtsumstände und des familiären Hintergrundes während der Entwicklung gibt es tendenzielle Unterschiede (nichteheliche Geburt forensische Patienten: 29,4\%, psychiatrische Patienten: 16,1\%).

Die Familienstrukturen während des Heranwachsens unterscheiden sich tendenziell (s. Abb. 22). 11,9\% der forensischen Patienten wuchsen ohne festen Wohnsitz auf, dies erlebte keiner der psychiatrischen Patienten. 


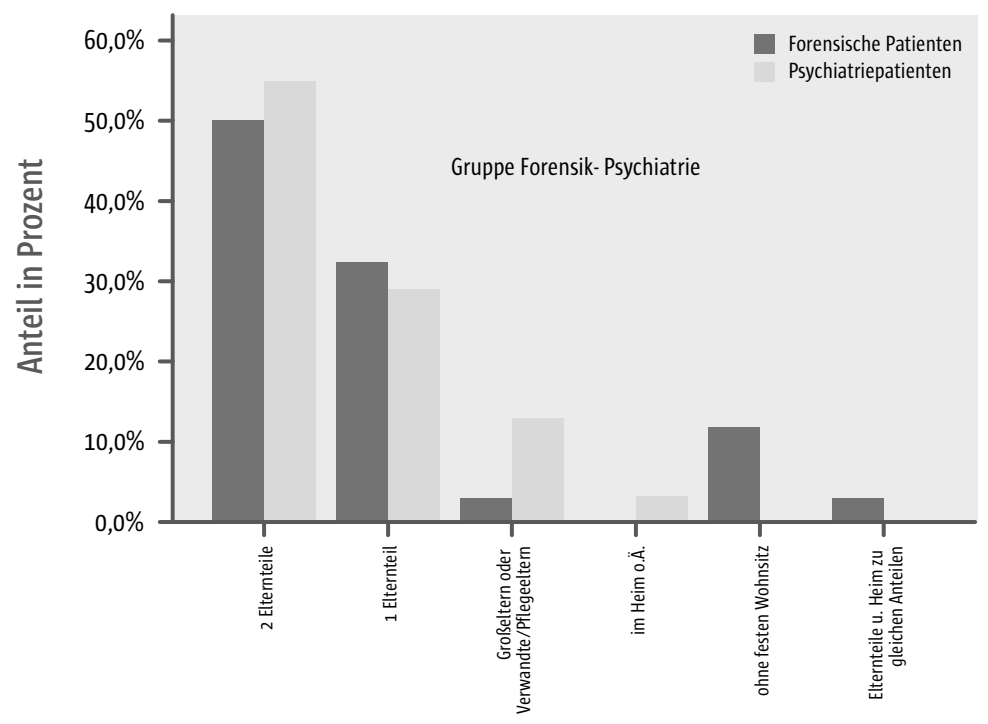

Abb. 22 Familienstrukturen während der Entwicklung (bis 15. Lebensjahr)

Bezüglich der Anzahl der Geschwister bzw. Halbgeschwister existieren keine signifikanten Unterschiede. Keine Geschwister hatten 8,8\% der forensischen Patienten und 9,7\% der psychiatrischen Patienten, ein bis drei Geschwister hatten $70,6 \%$ der forensischen und $71 \%$ der psychiatrischen Patienten und mehr als drei Geschwister ließen sich bei $20,6 \%$ der forensischen und 19,4\% der psychiatrischen Patienten feststellen.

Sexuellen Missbrauch erlebten 5,9\% der forensischen Patienten, jedoch keiner der Patienten der Psychiatrie, es gibt keinen signifikanten Unterschied.

Die Scheidungsanamnese der Eltern ist bei beiden Patientengruppen relativ ähnlich. 52,9\% der forensischen Patienten erlebten keine Scheidung der Eltern, 23,5\% widerfuhr sie im Alter von o bis fünf Jahren und bei $23,5 \%$ trat sie im Alter von fünf bis fünfzehn Jahren auf. Von den psychiatrischen Patienten wuchsen 53,3\% ohne Scheidung der Eltern auf, 30\% erlebten diese im Alter von o bis fünf und 16,7\% im Alter von fünf bis fünfzehn.

Die Berufe der Eltern sind in den Abbildungen 23 und 24 aufgeführt. Die Eltern der psychiatrischen Patienten hatten deutlich höhere Bildungsstände.

In der Beziehung der Patienten zur Mutter zeigen sich tendenzielle Unterschiede, da 41,2\% der forensischen und 22,6\% der psychiatrischen Patienten über eine gestörte Beziehung zur Mutter berichteten.

Delinquentes Verhalten in der Familie wurde bei 5,9\% der forensischen und 3,2\% der psychiatrischen Patienten angegeben, damit ergeben sich keine signifikanten Unterschiede.

In der Befragung zum sozialen Umfeld wurden folgende Angaben erhoben. 


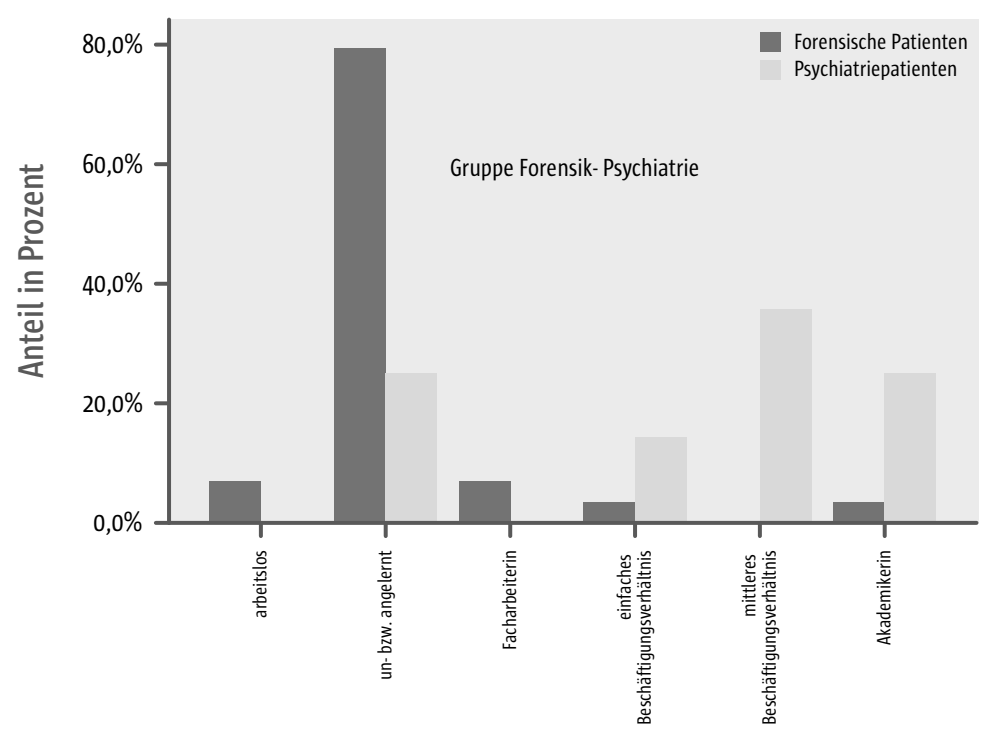

Abb. 23 Beruf der Mutter

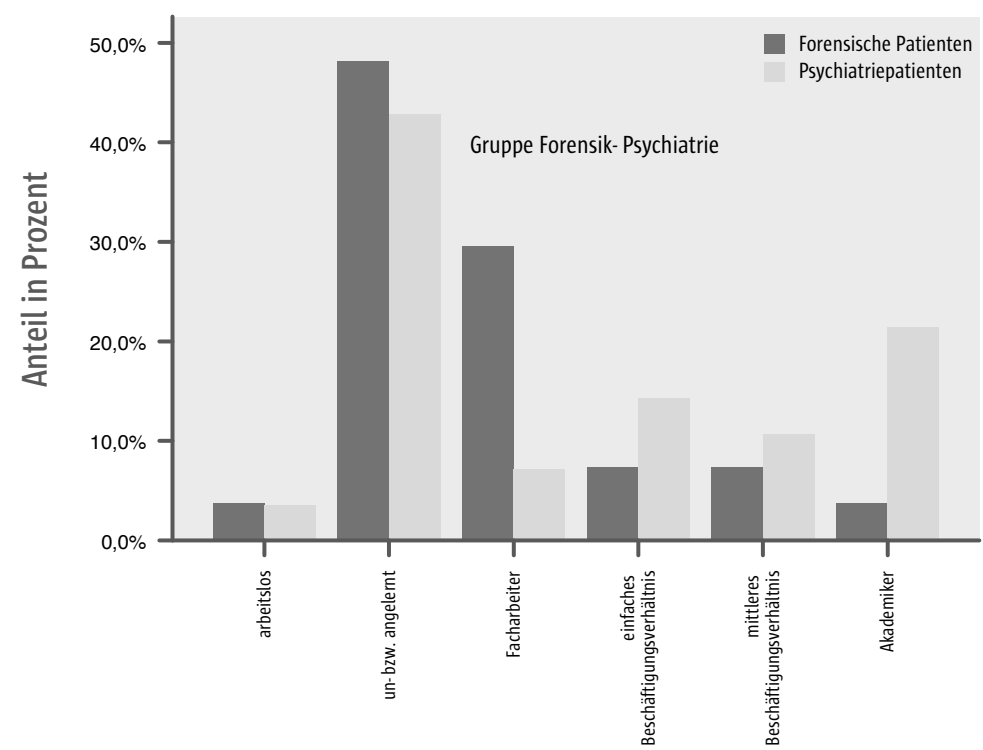

Abb. 24 Beruf des Vaters

Unterschiede in der Qualität der Freundschaften in der Kindheit bzw. frühen Jugend sind nicht vorhanden, der Großteil der Patienten sah sich selbst sogar als recht beliebt (s. Abb. 25).

Bei der wohnlichen Situation gibt es kaum Unterschiede zwischen den beiden Gruppen. 76,5\% der forensischen Patienten besaßen eine eigene Woh- 
nung, im betreuten Einzelwohnen befanden sich 3\%, 8,8\% wohnten bei Freunden, $8,8 \%$ waren noch bei den Eltern oder Großeltern untergebracht und 3\% waren ohne festen Wohnsitz. Bei den psychiatrischen Patienten besaßen 74, $2 \%$ eine eigene Wohnung, 22,6\% lebten noch bei ihren Eltern oder Großeltern und $3,2 \%$ waren ohne festen Wohnsitz. Im betreuten Wohnen oder bei Freunden hielt sich keiner der psychiatrischen Patienten auf.

Der Familienstand unterscheidet sich bei beiden Gruppen nicht signifikant. 6,5\% der psychiatrischen Patienten waren zum Zeitpunkt der Untersuchung verheiratet und lebten zusammen. Alle anderen Patienten beider Cruppen waren entweder geschieden, getrennt lebend oder ledig.

Im Rahmen einer bestehenden Partnerschaft gibt es keine Unterschiede, $35 \%$ beider Cruppen lebten zum Untersuchungszeitpunkt in einer Beziehung. Tendenzielle Unterschiede traten dagegen in der Qualität der Partnerschaft auf. Nur $50 \%$ der forensischen Patienten mit einem Partner schilderten ihre Beziehung als gut, wogegen $81,8 \%$ der psychiatrischen Patienten kaum Probleme hatten.

In Bezug auf eigene Kinder zeigten sich tendenzielle Unterschiede zwischen beiden Cruppen, da nur 20,6\% der forensischen Patienten, aber 32,3\% der psychiatrischen Patienten Kinder hatten.

In der Suchtanamnese gibt es tendenzielle Unterschiede beim Alkoholkonsum. 91, $2 \%$ der forensischen Patienten tranken Alkohol, hiervon $67,7 \%$ regelmäßig ( $61,8 \%$ der Gesamtgruppe der forensischen Patienten). Von den psychiatrischen Patienten tranken 73,3\% Alkohol, davon 45,5\% regelmäßig (33,3\% der Gesamtgruppe der psychiatrischen Patienten).

In der Art und Anzahl der unterschiedlichen Drogen gibt es beträchtliche Differenzen (Mann-Whitney-U-Test). Die Patienten der Forensik, die Erfahrungen mit Drogen hatten, konsumierten im Durchschnitt 4,2 verschiedene Drogentypen, die psychiatrischen Patienten 2,7 (s. Abb. 26).

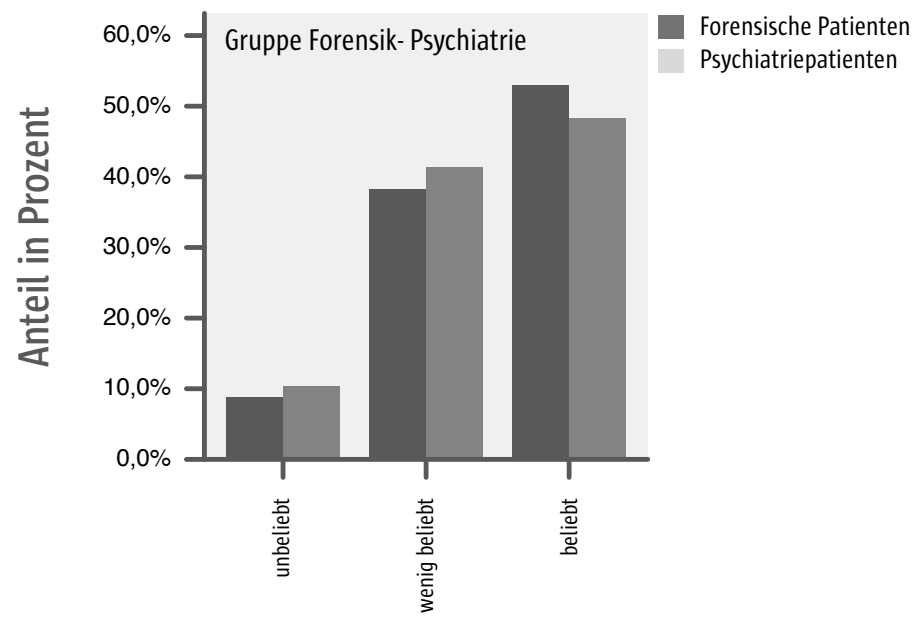

Abb. 25 Soziale Kontakte 


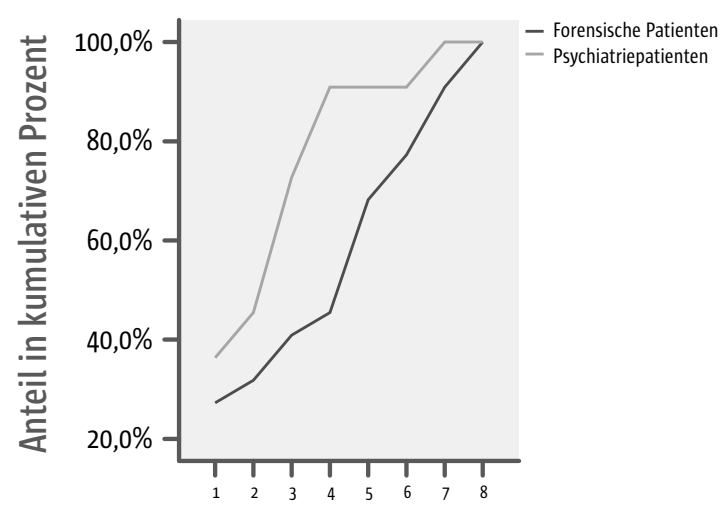

Abb. 26 Anzahl der konsumierten Drogentypen

Bei den Patienten, die anamnestisch Drogen genommen hatten, überwog in beiden Cruppen der Konsum so genannter weicher Drogen wie Cannabis (100\% in der Forensik und 90,9\% in der Psychiatrie). 63,6\% der forensischen Patienten gaben an, Designerdrogen konsumiert zu haben, während es in der Psychiatrie nur 45,5\% waren. Halluzinogene nahmen im Vorfeld 54,5\% der forensischen und 45,5\% der psychiatrischen Patienten, Amphetamine und andere Stimulanzien 45,5\% (Forensik) bzw. 36,4\% (Psychiatrie), Benzodiazepine und andere Sedativa $36,4 \%$ der forensischen und 27,3\% der psychiatrischen Patienten. Andere Psychopharmaka hatten 18,2\% (Forensik) und 9,1\% (Psychiatrie) genommen.

Ein polytoxikomanes Suchtverhalten (Konsum von drei oder mehr verschiedenen Drogentypen) zeigten 68,3\% der forensischen und 54,5\% der psychiatrischen Patienten. Es bestehen hier keine signifikanten Unterschiede.

An komorbiden psychischen Störungen lag vor allem ein Suchtmittelabusus bei den Patienten der Forensik vor, häufig in Kombination mit Persönlichkeitsstörungen und Verhaltensstörungen. Nur bei zwei der Patienten konnte kein Suchtmittelabusus im Sinne von Alkohol- oder Drogenkonsum eruiert werden. Allerdings waren diese Patienten nach $\$ 6_{3}$ StGB in der forensischen Klinik untergebracht. Dennoch waren beide Patienten Raucher.

Signifikante Unterschiede zeigen sich in Bezug auf die Komorbidität von Persönlichkeitsstörungen. 47,1\% der forensischen Patienten litten zum Untersuchungszeitpunkt unter einer Persönlichkeitsstörung, von den psychiatrischen Patienten waren es nur 19,4\%. Tendenzielle Unterschiede gibt es im Zusammenhang mit dissozialen Persönlichkeitsstörungen. Bei 37,5\% der forensischen Patienten konnte diese Diagnose gestellt werden, welche bei keinem der psychiatrischen Patienten zu eruieren war. Im Bereich der affektiven Erkrankungen gab es keine Unterschiede, 17,6\% der forensischen Patienten und 12,9\% der psychiatrischen Patienten litten unter einer affektiven Störung. Die Abbildung 27 zeigt einen Überblick über die komorbide Erkrankungen. 


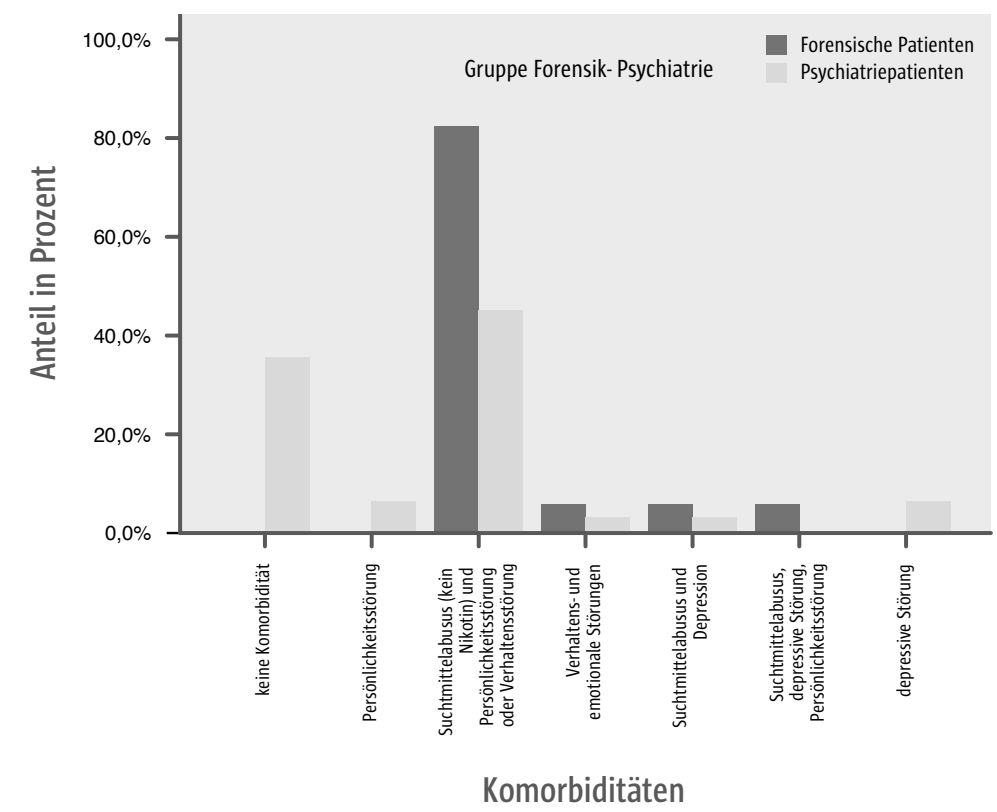

Abb. 27 Komorbiditäten

Die Deliktspanne der forensischen Patienten zeigt Abbildung 28. Deutlich überwiegen die sogenannten Gewaltdelikte, aber es gab auch zum Zeitpunkt der Untersuchung sieben Sexualdelinquenten. Bei fünf von ihnen zeigten sich eindeutig Symptome aus dem Krankheitsspektrum einer Aufmerksamkeitsdefizit-/Hyperaktivitätsstörung. Vier dieser Patienten waren Mehrfachtäter im Bereich der Sexualdelikte, bei drei der Mehrfachdelinquenten war die ADHS sehr stark ausgeprägt (s. Abb. 29).

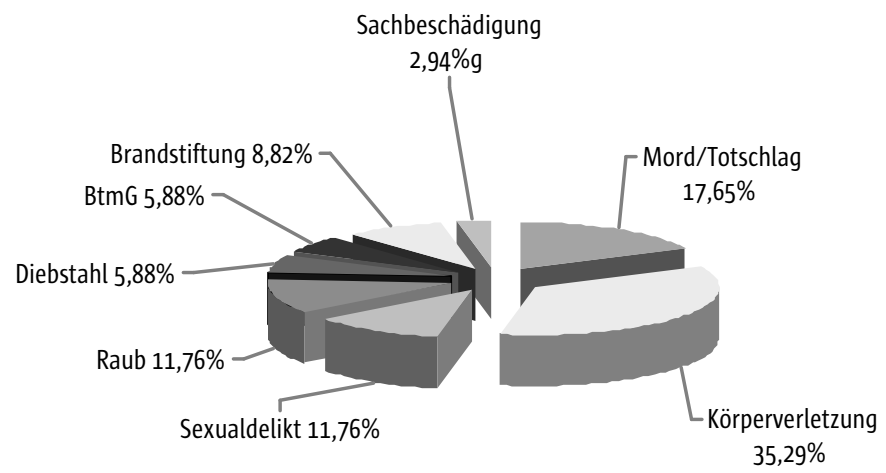

Abb. 28 Anlassdelikt 


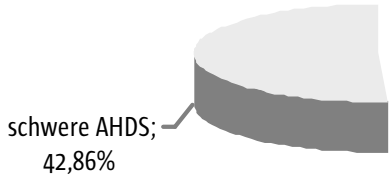

$42,86 \%$

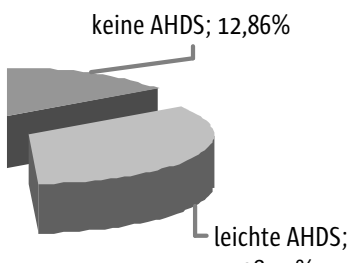

$28,57 \%$

Abb. 29 Ausprägungsgrad der ADHS bei Sexualdelinquenten

\subsection{Diskussion}

In unserer Untersuchung wurden Selbstbeurteilungsskalen als zentrales Diagnostikinstrument eingesetzt, auf deren Grundlage eine weitere Diagnostik erfolgte.

Problematisch kann sich dabei ein Antwortstil im Sinne sozialer Erwünschtheit auf die Qualität auswirken. Daneben ist eine Bejahungstendenz zu beachten (Ziegler 2003). Zusätzlich wurde der Cut-Off in den Fragebögen herabgesetzt. Dies geschah in dem Wissen, dass bei einer angenommenen Prävalenz einer ADHS von ca. $20 \%$ in forensischen Populationen in einer Klinik mit ungefähr 70 Patienten bei strengen Kriterien nur eine sehr kleine Patientengruppe zur Verfügung stehen würde und dass die rückwirkende Beantwortung ohne Fremdanamnese Fehler implizieren würde (falsch negative Ergebnisse). Allerdings muss der Verschmälerung der Diagnostik hierbei Rechnung getragen werden, wir sprechen deshalb von Symptomen aus dem Spektrum der ADHS. Des Weiteren erreichte ein Teil der Patienten zwar den Cut-Off in den Testbögen, zeigte aber klinisch kein vollständiges Bild einer ADHS. Dies deutet auf eine Teilpersistenz der Symptomatik hin.

In der Diagnostik war außerdem das vom DSM-IV geforderte Vorliegen von Symptomen einer ADHS in der Kindheit, wenn die Diagnose einer ADHS im Erwachsenenalter gestellt werden soll, problematisch. Es zeigte sich, dass bei mehreren Patienten dieses Kriterium mittels WURS nicht erfüllt wurde und trotzdem eine Symptomatik im Erwachsenenalter (nach ADHS-SB) vorlag. Dies verdeutlicht noch einmal die besondere Bedeutung des herabgesetzten CutOffs in der WURS (von 9o auf 80 Punkte). Eine reine Erwachsenensymptomatik war auch bei Verwendung des „normalen“ Cut-Offs (15 Punkte) in der ADHS-SB nachzuweisen und nicht nur beim reduzierten Cut-Off (1o Punkte). In mehreren Fällen waren dementsprechend trotz Herabsetzen des Cut-Offs keine Symptome in der Kindheit zu eruieren, sondern nur im Erwachsenenalter. Symptome in der Kindheit waren jedoch trotz starker Ausprägung der Erkrankung im Erwachsenenalter nicht in allen Fällen zu eruieren. Dieses Ergebnis erfordert für nachfolgende Studien zur ADHS ein breiteres Screening sowie eine eventuelle Ausweitung der diagnostischen Kriterien. Ferner könnte es helfen, bei der Entwicklung neuer Skalen auf eine gerade Anzahl von 
Antwortmöglichkeiten zu achten. So entfällt die bequeme Art, sich für die Mitte zu entscheiden.

Im Bereich des TCI zeigten beide Gruppen ähnliche Ergebnisse. Patienten mit starkem Neugierverhalten haben häufig auch Probleme mit ihrer Impulsivität (Edel und Vollmoeller 2006). Dies bestätigte sich in diesem Test, in beiden Patientengruppen war das Neugierverhalten recht stark ausgeprägt.

Bei den erfassten Unterschieden in den Persönlichkeitstendenzen haben psychiatrische Patienten eher eine antisoziale oder passiv-aggressive Temperamentstruktur, forensische Patienten deutlichere explosive Strukturen; ein sozial erwünschter Antwortstil muss aber beachtet werden. Im Rahmen der Unterbringung in der forensischen Klinik versuchen die Patienten bewusst oder unbewusst Antworten zu geben, die sie im Zusammenhang mit therapeutischen Erwartungen und Lockerungsmaßnahmen sehen. Die hohen Werte im Bereich des explosiven Verhaltens liegen der Impulsivität der ADHS zu Grunde.

Die Ergebnisse der IQ-Testung zeigen für die psychiatrische Gruppe, dass eine ADHS nicht zwangsläufig mit niedrigerer Intelligenz einhergehen muss. Bei entsprechender Förderung, Unterstützung und Bildung können auch diese Patienten eine hohe Intelligenz ausbilden. Dieser Zusammenhang entspricht auch den Ergebnissen einer Studie von Palacios und Semrud-Clikeman (2005), die bei Patienten mit alleiniger ADHS einen etwa gleich hohen IQ-Wert fanden wie in der gesunden Kontrollgruppe (104 vs. 103). Erst bei komorbidem Vorliegen einer oppositionellen Verhaltensstörung zeigten sich deutlich niedrigere IQ-Werte, entsprechend unserem Ergebnis der forensischen Gruppe.

Bei Patienten mit einer ADHS kommt es aufgrund der spezifischen Symptomatik häufiger zu Konflikten mit der Umwelt als bei gesunden Menschen (Wender 200o). Diese können auf verschiedenen Wegen zu späteren delinquenten Verhaltensweisen führen. Im familiären Gefüge lösen eigensinnige, unlenkbare und störrische Kinder elterlichen Stress aus, welcher zum Teil zu ungünstigen Erziehungsmethoden (Schläge, Arreste, häufige lautstarke Auseinandersetzungen) führen kann. Dem Kind wird dabei das Erlernen von Kontrolle über sein eigenes Verhalten versagt. Der ungünstige Erziehungsstil verstärkt als Circulus Vitiosus die Erziehungsschwierigkeiten, häufig kommen schulische Probleme und Lernschwierigkeiten mit hinzu. Dies führt wiederum zu Schulschwänzen oder frühzeitigem Schulabbruch sowie einer mangelnden Schulbildung. Es kommt zum Anschluss an „Gleichgesinnte“ und in diesen Gruppen werden delinquentes Verhalten und Alkohol- oder Drogenmissbrauch praktiziert. Dass dies alles auch wieder zu weiteren Schwierigkeiten in der elterlichen Erziehung führt, ist abzusehen. Durch mangelnde Bildung kommt es zu Problemen in Ausbildung oder Beruf, zusätzlich fördert die Symptomatik der ADHS Abbrüche oder Kündigungen. Letztendlich besteht die Gefahr der Entwicklung eines antisozialen Verhaltens, welches im Weiteren in die Delinquenz mündet (McMurran 2001).

Im Gegensatz dazu zeigte sich in der vorliegenden Studie jedoch bei vielen Patienten mit ADHS, dass die beschriebenen Endpunkte nicht notwendigerweise im Zusammenhang mit einer ADHS erreicht werden müssen. Ungüns- 
tige Familienverhältnisse und Erziehungsstile, vor allem familiäre Gewalt, entwickeln sich nicht allein im Zusammenhang mit einer ADHS. Viele der psychiatrischen Patienten hatten, im Gegensatz zu den forensischen Patienten, keine familiären Schwierigkeiten. Allerdings sieht es so aus, als ob diese Probleme die delinquente Entwicklung im Zusammenhang mit einer ADHS fördern. Ein Croßteil der forensischen Patienten wuchs in ungeordneten familiären Verhältnissen auf, war Scheidungskind und erfuhr wenig Zuwendung durch die Eltern. Viele berichteten über eine gestörte Beziehung zu den Eltern, welche nach Freidenfelt und af Klinteberg (2003) dissoziale Verhaltensweisen fördert. Auch der Bildungsstand und eine evtl. Berufstätigkeit der Eltern waren bei den forensischen Patienten deutlich schlechter. Eine alleinige ADHS, in Kombination mit guten familiären und sozialen Verhältnissen, stellt nach den Ergebnissen unserer Untersuchung keine Gefahr für den weiteren Lebensweg dar. Erst familiäre und soziale Konflikte, welche bei den Patienten der Forensik gehäuft anzutreffen waren, beeinträchtigen den weiteren Werdegang.

Der Relevanz dieser Ergebnisse sollte in der Behandlung der ADHS Beachtung geschenkt werden. Eine gezielte Förderung von benachteiligten Kindern beeinflusst die Entwicklung positiv und kann späterer Straffälligkeit vorbeugen.

Interessanterweise gab keine von beiden Cruppen deutlich gestörte Freundschaften in der Kindheit an, sie selbst hatten zum Großteil den Eindruck, beliebt zu sein. Dies steht im Gegensatz zu den vielfach beschriebenen unbeständigen und problembehafteten sozialen Kontakten (Wender 200o; Krause und Krause 2003; Schneider und Rösler 2005). Auch bestehende Partnerschaften wurden in mehr als der Hälfte der Fälle als gut beschrieben. Hierbei sei jedoch darauf hingewiesen, dass nur die aktuelle Beziehung berücksichtigt wurde, wie häufig es in der Vergangenheit zu Partnerwechseln aufgrund von Konflikten kam, ist nicht untersucht worden.

Die Ergebnisse zeigen also, dass eine ADHS nicht zwangsläufig mit einer delinquenten Entwicklung in Zusammenhang gebracht werden sollte. Patienten, deren familiäre Strukturen geordnet waren, zeigten kaum straffällige Tendenzen. In dieser Cruppe waren auch die Komorbiditäten Verhaltens- und Persönlichkeitsstörungen deutlich geringer, ebenso wie generell ungünstige Sozialisationsparameter und Drogenabusus.

Das erhöhte Delinquenzrisiko bei Erwachsenen mit ADHS steht in Zusammenhang mit einer zusätzlich vorhandenen Persönlichkeitsstörung, vor allem im Rahmen einer dissozialen Persönlichkeitsstörung oder einer Verhaltensstörung. Laut aktueller Studienlage ist bei $18 \%$ bis $23 \%$ der Jungen mit ADHS im späteren Erwachsenenalter eine komorbide Störung im Sinne einer dissozialen Persönlichkeitsstörung zu finden (Sobanski 2006). In unserer Studie konnte bei 47,1\% der forensischen (insbesondere dissoziale Persönlichkeitsstörungen) und 19,4\% der psychiatrischen Patienten eine komorbide Persönlichkeitsstörung diagnostiziert werden. Deutlich wird, dass nicht die ADHS die Ursache späterer Delinquenz zu sein scheint, sondern komorbide Verhaltens- und Persönlichkeitsstörungen sowie Missverhältnisse in der Entwicklung ausschlaggebend sind. In der Literatur zeigte sich bei allen Untersuchun- 
gen zur generellen psychischen Komorbidität von Straftätern bei den Patienten primär eine Störung des Sozialverhaltens, zum Teil mit einer ADHS kombiniert (Haapasalo und Hämäläinen 1996; Rayner et al. 2005; Hollander und Turner 1985; Timmons-Mitchell et al. 1997). Frühzeitiges Erkennen von komorbiden Verhaltens- und dissozialen Persönlichkeitsstörungen ist demnach wichtig, um delinquenter Entwicklung rechtzeitig vorbeugen zu können.

Loeber et al. (1995) und Lie (1992) kamen zu dem Schluss, dass eine Verhaltensstörung oder antisoziale Persönlichkeitsstörung zu späterer Delinquenz führt, nicht die ADHS. Allerdings beeinflusst eine ADHS eine Verhaltens- und Persönlichkeitsstörung negativ und führt zu einem früheren Ausbruch, wodurch der Weg in die Delinquenz erleichtert wird (Thompson et al. 1996). Warum allerdings dissoziale Persönlichkeitsstörungen und Verhaltensstörungen im Zusammenhang mit einer ADHS häufiger auftreten, ist noch nicht befriedigend geklärt. Nach ICD-10 stellt eine ADHS kombiniert mit einer Störung des Sozialverhaltens einen Subtyp der Erkrankung dar, welcher mit Verhaltensauffälligkeiten und einer erhöhten Wahrscheinlichkeit für delinquentes Verhalten im Erwachsenenalter einhergeht. Es kommt zu Überschneidungen bei den sozialen Problematiken, die bei Verhaltensstörungen und Aufmerksamkeitsstörungen auftreten (Windle 1993). Beide Erkrankungen scheinen miteinander verwandt zu sein (Vollmoeller und Edel 2006). Sowohl die ADHS als auch die Persönlichkeitsstörung beginnen in jungen Jahren und beide sind zeitlich relativ überdauernd bis persistierend. Sie treten vielseitig und situationsübergreifend auf, Auffälligkeiten sind in mehreren psychischen Bereichen zu erkennen und die soziale und berufliche Leistungsfähigkeit der Betroffenen wird beträchtlich eingeschränkt. Bei beiden Krankheiten wird von einer multifaktoriellen Vererbung ausgegangen. Hyperaktivität, Impulsivität und kindliche Verhaltensstörungen bilden den Ausgangspunkt einer Verhaltensweise, welche zu antisozialen Persönlichkeitstendenzen in der Adoleszenz führt.

Im Zusammenhang mit der Suchtanamnese zeigten sich in der vorliegenden Untersuchung bezüglich der Anzahl der konsumierten Drogen deutliche Unterschiede. Der hohe Anteil eines Substanzkonsums in beiden Untersuchungsgruppen findet sich auch in anderen Studien. Biederman et al. (1995) beschrieben einen Substanzmissbrauch bei 30-50\% der Patienten mit einer ADHS. In unserer forensischen Cruppe ließen sich bei ungefähr der Hälfte der ursprünglich gescreenten suchtkranken (und delinquenten) Patienten Symptome einer ADHS belegen. Dies deckt sich mit anderen Studien, in denen deutlich erhöhte Prävalenzraten einer ADHS bei alkoholabhängigen oder drogensüchtigen Patienten gefunden wurden (Kalbag und Levin 2005). Bis zu einem Drittel der erwachsenen Kokainabhängigen leiden unter einer komorbiden ADHS (Johann et al. 2003).

Loeber et al. (1999) betonten jedoch, dass eine vorliegende ADHS nicht die Persistenz eines Drogen- bzw. Substanzabusus ins Erwachsenenalter beeinflusst. Stattdessen korreliert ein persistierender Missbrauch deutlich mit bestehenden delinquenten Verhaltensweisen. Biederman et al. (1995) kamen zu 
dem Ergebnis, dass Drogenabusus als Bestandteil delinquenten Verhaltens signifikant häufiger bei Vorliegen einer antisozialen Persönlichkeitsstörung vorkommt, unabhängig vom Ausprägungsgrad einer ADHS. Das Suchtverhalten ist bei Komorbidität von ADHS und Störung des Sozialverhaltens ausgeprägter (früherer Beginn, größere Anzahl von konsumierten Drogen) als bei alleiniger ADHS (Thompson et al. 1996). Dies konnten auch wir bestätigen, unter den delinquenten Patienten mit Persönlichkeits- und Verhaltensstörungen war ein deutlich höherer Alkohol- und Drogenkonsum anzutreffen.

Studien belegen, dass die Behandlung von Kindern mit ADHS mittels Stimulanzien eine protektive Wirkung hinsichtlich einer späteren Entwicklung von Suchterkrankungen hat (Davids und Gastpar 2003). In diesem Kontext sollte die Therapie der ADHS also frühzeitig erfolgen. Des Weiteren lassen sich hierdurch auch Störungen des Sozialverhaltens positiv beeinflussen (Retz et al. 2007). Wenn bei süchtigen Patienten, die gemäß $₫ 64$ StGB untergebracht sind, als Komorbidität eine ADHS vorliegt, sollte diese mitbehandelt werden, um die Sucht selbst positiv beeinflussen zu können. In forensischen Kliniken wird wegen der Missbrauchsgefahr (Handeltreiben) in der Regel auf nicht stimulierende Präparate ausgewichen.

Die in unserer Untersuchung ermittelte Prävalenz von ADHS-assoziierten Symptomen in einer forensischen Population betrug $65,7 \%$. Dies steht in Einklang mit einigen nationalen und internationalen Studien, die eine signifikant höhere Anzahl von ADHS-Erkrankten in forensischen Populationen fanden (Dalteg und Levander 1998; Rösler et al.; 2004; Timmons-Mitchell et al. 1997; Ziegler et al. 2003).

Ebenso zeigten sich im Bereich der Sexualdelinquenz Zusammenhänge zwischen einer ADHS und sexuell auffälligen bzw. straffälligen Verhaltensweisen. Es gibt erste Studien, die zeigen konnten, dass ein Großteil der Sexualstraftäter unter einer kindlichen bzw. persistierenden ADHS litten bzw. leiden (Blocher et al. 2001; Giotakis et al. 2005; Vaigh-Koch et al. 2001). Wenngleich wir nur eine sehr kleine Stichprobe von Sexualdelinquenten auf das Vorliegen einer ADHS untersuchen konnten, kommen wir zu einer starken Belastung dieser Täter mit ADHS, insbesondere bei Wiederholungstätern.

Worin jedoch die Ursachen der deutlich erhöhten Prävalenzen innerhalb delinquenter Cruppen zu sehen sind, bleibt unklar. Das Risiko für Festnahmen und Inhaftierungen scheint bei Patienten mit einer ADHS deutlich erhöht zu sein (Rasmussen und Gillberg 2000). Allerdings sei noch einmal auf die bereits erwähnte Komorbidität einer dissozialen Persönlichkeitsstörung bei delinquenten ADHS-Patienten hingewiesen. Dieser Zusammenhang wurde bisher kaum beachtet, unsere Resultate beweisen jedoch, dass diese Komorbidität einen wichtigen Entwicklungsfaktor darstellt. So fanden auch Rey et al. (2005) bei der Untersuchung von aggressiven und delinquenten Jugendlichen eine erhöhte Prävalenz der ADHS im Vergleich zur Normalbevölkerung, bei genauerer Untersuchung der Daten kamen die Autoren jedoch zu dem Ergebnis, dass nicht die kindliche ADHS zu späterer aggressiver Delinquenz führt, sondern diese ein Ergebnis von aggressivem Verhalten in der Kindheit ist. Bestehendes 
antisoziales Verhalten ist der Prädiktor für eine spätere Delinquenz und nicht die alleinige Erkrankung mit einer ADHS, Patienten mit alleiniger ADHS und keinen komorbiden Verhaltens- und Persönlichkeitsstörungen haben kein erhöhtes Delinquenzrisiko. Bei gemeinsamem Auftreten dieser beiden Störungen ist das Risiko einer delinquenten Entwicklung im Vergleich zu Patienten mit alleiniger Verhaltens- oder dissozialen Persönlichkeitsstörung jedoch deutlich erhöht (Lie 1992). In unserer Patientengruppe der Allgemeinpsychiatrie litt keiner unter einer entsprechenden Komorbidität und es waren keine Konflikte mit dem Gesetz in dieser Gruppe zu eruieren. Eine entsprechende frühzeitige Intervention und Therapie beider Erkrankungen würde das Risiko einer delinquenten, antisozialen Entwicklung senken (Foley et al. 1996; Fergusson et al. 1993).

\section{Literatur}

American Psychiatric Association Diagnostisches und statistisches Manual psychischer Störungen: DSM-IV. Göttingen: Hogrefe, Verlag für Psychologie (1998).

Barkley, RA, Fischer, M, Edelbrock, CS, Smallish, L.The adolescent outcome of hyperactive children diagnosed by research criteria: I. An 8-year prospective follow-up study. I Am Acad Child Adolesc Psychiatr. 1990 |ul; 29(4): 546-57.

Biederman, J, Faraone, SV, Spencer, T.Patterns of psychiatric comorbidity, cognition and psychosocial functioning in adults with attention deficit hyperactivity disorder. Am J Psychiatr. 1993 Dec; 150(12): 1792-1798.

Biederman, I, Wilens, T, Mick, E, Milberger, S, Spencer, TJ, Faraone, SV.Psychoactive substance use disorders in adults with attention deficit hyperactivity disorder (ADHD): effects of ADHD and psychiatric comorbidity. Am J Psychiatr. 1995 Nov; 152(11): 1652-1658.

Blocher, D, Henkel, K, Retz, W, Retz-Junginger, P, Thome, I, Rösler, M. Symptome aus dem Spektrum des hyperkinetischen Syndroms bei Sexualdelinquenten. Fortschr Neurol Psychiatr. 2001 0kt; 69(10): 453-459.

Cadoret, RI, Stewart, MA. An adoption study of attention deficit/hyperactivity/aggression and their relationship to adult antisocial personality. Compr Psychiatr. 1991 Jan-Feb; 32(1): 73-82.

Cloninger, CR, Przybeck, TR, Svrakic, DM, Wetzel, RD. Das Temperament- und Charakter-Inventar (TCI). Ein Leitfaden über seine Entwicklung und Anwendung (Übersetzung und Bearbeitung von Jörg Richter, Martin Eisemann, Gabriele Richter, C. Robert Cloninger). Frankfurt a. M.: Swets (1999).

Conners, CK, Erhardt, D, Sparrow, E. CAARS. Conners' Adult ADHD Rating Scales North Tonawanda, New York: MHS (1999).

Conners, CK, Erhardt, D, Epstein, JN, Parker, JDA, Sitarenios, G, Sparrow, E. Self-ratings of ADHD symptoms in adults: I. Factor structure and normative data. I Att Dis. 1999 Oct; 3(3): 141-151.

Dalteg, A, Levander, S. Twelve thousand crimes by 75 boys: A 20-year follow-up study of childhood hyperactivity. J Forens Psychiatr. 1998 May; 9(1): 39-57.

Davids, E, Gastpar, M. Aufmerksamkeitsdefizit-/Hyperaktivitätsstörung und Substanzmittelabhängigkeit. Psychiatr Prax. 2003 Mai; 30(4): 182-186.

Edel, MA, Vollmoeller, W. ADHS und Sucht. In: Edel, MA, Vollmoeller, W [Hrsg] Aufmerksamkeitsdefizit-/Hyperaktivitätsstörung bei Erwachsenen. Heidelberg: Springer (2006): 104-132.

Faraone, SV, Biederman, I. Neurobiology of attention-deficit hyperactivity disorder. Biol Psychiatr. 1998 Nov 15; 44(10): 951-958.

Faraone, SV, Spencer, T, Aleardi, M, Pagano, C, Biederman, I. Meta-analysis of the efficacy of methylphenidate for treating adult attention-deficit/hyperactivity disorder. I Clin Psychopharmacol. 2004 Feb; 24(1): 24-29.

Faraone, SV, Biederman, I, Mick, E, Williamson, S, Wilens, T, Spencer, T, Weber, W, Jetton, I, Kraus, I, Pert, I, Zallen, B. Family study of girls with attention deficit hyperactivity disorder. Am | Psychiatr. 2000 |ul; 157(7): 1077-1083. 
Faraone, SV, Perlis, RH, Doyle, AE, Smoller, JW, Goralnick, II, Holmgren MA, Sklar, P. Molecular genetics of attention-deficit/hyperactivity disorder. Biol Psychiatr. 2005 Jun 1; 57(11): 1313-1323.

Farrington, DP. Adolescent violence: Findings and implications from the Cambridge Study. In: Boswell, $G$ [Hrsg].

Violent children and adolescents: Asking the question why. Philadelphia: Whurr Publishers (2000): 19-35.

Fergusson, DM, Horwood, L), Lynskey, MT. The effects of conduct disorder and attention deficit in middle childhood on offending and scholastic ability at age 13. I Child Psychol Psychiatr. 1993 Sep; 34(6): 899-916.

Foley, HA, Carlton, CO, Howell, RJ. The relationship of attention deficit hyperactivity disorder and conduct disorder to juvenile delinquency: legal implications. Bull Am Acad Psychiatr Law. 1996; 24(3): 333-345.

Freidenfelt, I, af Klinteberg, B. Are negative social and psychological childhood characteristics of significant importance in the development of psychosocial dysfunctioning? Intern J Forens Ment Health. 2003 Fal; 2(2): 181-193.

Giotakos, 0, Markianos, M, Vaidakis, N. Aggression, impulsivity, and plasma sex hormone levels in a group of rapists, in relation to their history of childhood attention-deficit/hyperactivity disorder symptoms. I Forens Psychiatr Psycholog. 2005 Jun; 16(2): 423-433.

Gittelman, R, Mannuzza, S, Shenker, R, Bonagura, N. Hyperactive boys almost grown up: I. Psychiatric status. Arch Gen Psychiatr. 1985 Oct; 42(10): 937-947.

Groß, I, Blocher, D, Trott, GE, Rösler, M. Erfassung des hyperkinetischen Syndroms bei Erwachsenen. Nervenarzt. 1999 Jan; 70(1): 20-25.

Haapasalo, I, Hämäläinen, T. Childhood family problems and current psychiatric problems among young violent and property offenders. I Am Acad Child Adolesc Psychiatr. 1996 Oct; 35(10): 1394-1401.

Hollander, HE, Turner, FD. Characteristics of incarcerated delinquents: relationship between development disorders, environmental and family factors, and patterns of offense and recidivism. I Am Acad Child Psychiatr. 1985 Mar; 24(2): 221-226.

Johann, M, Bobbe, G, Putzhammer, A, Wodarz, N. Comorbidity of alcohol dependence with attention-deficit hyperactivity disorder: differences in phenotyp with increased severity of the substance use disorder, but not in genotype (serotonin transporter and 5-hydroxytryptamine-2c receptor). Alcohol Clin Exp Res. 2003 Oct; 27(10): 1527-1534.

Kalbag, AS, Levin, FR. Adult ADHD and substance abuse: diagnostic and treatment issues. Subst Use Misuse. 2005; 40(13-14): 1955-81, 2043-8.

König, S, Wodarz, N, Unglaub, W, Johann, M. Aufmerksamkeitsdefizit/Hyperaktivitätssyndrom (ADHS) bei erwachsenen Drogenabhängigen. Psychiatr Praxis. 2007 Jan; 34 (Suppl 1): 71-72.

Krause, J. Diagnostik und Therapie der ADHS im Erwachsenenalter. In: Edel, MA, Vollmoeller, W [Hrsg] Aufmerksamkeitsdefizit-/Hyperaktivitätsstörung bei Erwachsenen. Heidelberg: Springer (2006): 30-48.

Krause, I, Krause, KH. ADHS im Erwachsenenalter. Stuttgart: Schattauer (2003).

Lie, N. Follow-ups of children with attention deficit hyperactivity disorder (ADHD). Review of literature. / Att Dis. 1996 Oct; 1(3): 147-161.

Murphy, K, Barkley, RA. Attention deficit hyperactivity disorder adults: comorbidities and adaptive impairments. Compr Psychiatr. 1996 Nov-Dec; 37(6): 393-401.

Ohlmeier, M, Peters, K, Buddensiek, N, Seifert, I, Wildt, B, Emrich, HM, Schneider, U. ADHS und Sucht. Psychoneuro. 2005; 31(11): 554-562.

Palacios, ED, Semrud-Clikeman, M. Delinquency, hyperactivity, and phonological awareness: a comparison of adolescents with ODD and ADHD. Appl NeuropsycActa Psychiatr Scand Suppl. 1992; 368: 1-40.

Loeber, R, Green, SM, Keenan, K, Lahey, BB. Which boys will fare worse? Early predictors of the onset of conduct disorder in a six-year longitudinal study. I Am Acad Child Adolesc Psychiatr. 1995 Apr; 34(4): 499-509.

Loeber, R, Stouthamer-Loeber, M, White, HR. Developmental aspects of delinquency and internalizing problems and their association with persistent juvenile substance use between ages 7 and 18. I Clin Child Psychol. 1999 Sep; 28(3): 322-332.

Lojewski, I, Wismann, B, Höger, C, Rothenberger, A, Havemann-Reinicke, U. Sind mit Methylphenidat therapierte Menschen einem erhöhten Missbrauchs- und Abhängigkeitsrisiko ausgesetzt? In: Am Abgrund. ADHS und Sucht ... Was nun? Fachbeiträge zum Thema: Aufmerksamkeits-Defizit-Syndrom. Forchheim: Bundesverband Aufmerksamkeitsstörung/Hyperaktivität e. V., (2002): 43-51. 
McMurran, M. Offenders with drink and Drug Problems. In: Hollin, C [Hrsg] Handbook of offender assessment and treatment Chichester: Wiley (2001): 481-493.

Murphy, K, Barkley, RA. Prevalence of DSM-IV symptoms of ADHD in adult licensed drivers: implications for clinical diagnosis. hol. 2005; 12(2): 94-105.

Rasmussen, P, Gillberg, C. Natural outcome of ADHD with developmental coordination disorder at age 22 years: a controlled, longitudinal, community-based study. I Am Acad Child Adolesc Psychiatr. 2000 Nov; 39(11): $1424-31$.

Rayner, I, Kelly, TP, Graham, F. Mental health, personality and cognitive problems in persistent adolescent offenders require long-term solutions: A pilot study. I Forensic Psychiatr Psychol. 2005 Jun;16(2): 248-262.

Retz, W, Retz-Junginger, P, Schneider, M, Scherk, H, Hengesch, G, Rösler, M. Suchtmittelgebrauch bei jungen erwachsenen Straftätern mit und ohne Aufmerksamkeitsdefizit-/Hyperaktivitätsstörung (ADHS). Fortschr Neurol Psychiatr. 2007 Mai; 75(5): 285-292.

Retz, W, Rösler, M. ADHS und Straffälligkeit. In: Edel, MA, Vollmoeller, W [Hrsg] Aufmerksamkeitsdefizit-/ Hyperaktivitätsstörung bei Erwachsenen. Heidelberg: Springer (2006): 134-152.

Retz-Junginger, P, Retz, W, Blocher, D, Weijers, HG, Trott, GE, Wender, PH, Rösler, M. Wender Utah Rating Scale (WURS-k). Die deutsche Kurzform zur retrospektiven Erfassung des hyperkinetischen Syndroms bei Erwachsenen. Nervenarzt. 2002 Sep; 73(9): 830-838.

Rey, IM, Sawyer, MG, Prior, MR. Similarities and differences between aggressive and delinquent children and adolescents in a national sample.

Aust N Z Psychiatr. 2005 May; 39(5): 366-372.

Rösler, M. Das hyperkinetische Syndrom im Erwachsenenalter. Psycho. 2001; 27(7): 380-384.

Rösler, M, Retz, W, Retz-Junginger, P, Hengesch, G, Schneider, M, Supprian, T,.

Schwitzgebel, P, Pinhard, K, Dovi-Akue, N, Wender, P, Thome, J. Prevalence of attention deficit-/hyperactivity disorder (ADHD) and comorbid disorders in young male prison inmates. Eur Arch Psychiatr Clin Neurosci. 2004 Dec; 254(6): 365-371.

Rösler, M, Retz, W, Retz-Junginger, P, Thome, I, Supprian, T, Nissen, T, Stieglitz, RD, Blocher, D, Hengesch, G, Trott, GE. Instrumente zur Diagnostik der Aufmerksamkeitsdefizit-/Hyperaktivitätsstörung (ADHS) im Erwachsenenalter. Selbstbeurteilungsskala (ADHS-SB) und Diagnosecheckliste (ADHS-DC). Nervenarzt. 2004 Sep; 75(9): 888-895.

Rösler, M, Retz, W, Thome, I, Schneider, M, Stieglitz, RD, Falkai, P. Psychopathological rating scales for diagnostic use in adults with attentiondeficit/hyperacti- vity disorder (ADHD). Eur Arch Psychiatr Clin Neurosci. 2006; 256 Suppl 1: 3-11.

Rubia, K, Overmeyer, S, Taylor, E, Brammer, M, Williams, SC, Simmons, A, Bullmore, ET. Hypofrontality in attention deficit hyperactivity disorder during higher-order motor control: a study with functional MRI. Am I Psychiatr. 1999 Jun; 156(6): 891-6.

Schneider, M, Rösler, M. Gibt es den erwachsenen Zappelphillip? extracta psychiatr/neurol 6/2005: 11-17.

Smidt, I, Heiser, P, Dempfle, A, Konrad, K, Hemminger, U, Kathöfer, A, Halbach, A, Strub, J, Grabarkiewicz, I, Kiefl, H, Linder, M, Knölker, U, Warnke, A, Remschmidt, H, Herpertz-Dahlmann, B, Hebebrand, J. Formalgenetische Befunde zur Aufmerksamkeitsdefizit-/Hyperaktivitätsstörung. Fortschr Neurol Psychiatr. 2003 Jul; 71(7): 366-377.

Sobanski, E. Psychiatric comorbidity in adults with attention-deficit/hyperactivity disorder (ADHD). Eur Arch Psychiatr Clin Neurosci. 2006; 256 Suppl 1: 26-31.

Sobanski, E. Psychiatrische Komorbidität bei Erwachsenen mit Aufmerksamkeitsdefizit-/Hyperaktivitätsstörung. Nervenheilkunde: Zeitschr interdiszipl Fortbild. 2006; 25(6), 430-436.

Sobanski, E, Alm, B. Retardiertes Methylphenidat. Eine Alternative in der medikamentösen Therapie bei erwachsenen Patienten mit Aufmerksamkeitsdefizit-/Hyperaktivitatsstörung. Nervenarzt. 2005 Nov; 76(11): 1412-1417.

Spencer, T, Biederman, I. Non-stimulant treatment for Attention-Deficit/Hyperactivity Disorder. I Att Dis. 2002; 6 Suppl 1: S109-19.

Timmons-Mitchell, I, Brown, C, Schulz, SC, Webster, SE, Underwood, LA, Semple, WE. Comparing the mental health needs of female and male incarcerated juvenile delinquents. Behav Scien Law. 1997 Spr; 15(2), 195-202. 
Thompson, LL, Riggs, PD, Mikulich, SK, Crowley, TJ. Contribution of ADHD symptoms to substance problems and delinquency in conduct-disordered adolescents. I Abnorm Child Psychol. 1996 Jun; 24(3): 325-347.

Todd, RD, Neuman, RJ, Lobos, EA, Jong, YJ, Reich, W, Heath, AC. Lack of association of dopamine D4 receptor gene polymorphisms with ADHD subtypes in a population sample of twins. Am / Med Genet. 2001 Jul 8; 105(5): 432-438.

Vaih-Koch, SR, Ponseti, I, Bosinkski, HAG. ADHD und Störung des Sozialverhaltens im Kindesalter als Prädiktoren aggressiver Sexualdelinquenz? Sexuologie. Vol 8(1) 2001, 1-18.

Vollmoeller W, Edel MA. ADHS und Persönlichkeit. In: Edel MA, Vollmoeller W [Hrsg] Aufmerksamkeitsdefizit-/ Hyperaktivitätsstörung bei Erwachsenen. Heidelberg: Springer (2006): 82-102.

Weltgesundheitsorganisation. Internationale Klassifikation psychischer Störungen: ICD-10. Kapitel V (F). Klinisch-diagnostische Leitlinien. Dilling, H, Mombour, W, Schmidt, MH [Hrsg.] Bern: Huber (2000).

Wender, PH. Themenschwerpunkt: Aufmerksamkeitsdefizit/Aktivitätsstörung im Erwachsenenalter. Psycho 2000, 26 (4):190-198.

Windle, M.A retrospective measure of childhood behavior problems and its use in predicting adolescent problem behaviors. I Stud Alcohol. 1993 Jul; 54(4), 422-431.

Zametkin, A], Liebenauer, LL, Fitzgerald, GA, King, AC, Minkunas, DV, Herscovitch, P, Yamada, EM, Cohen, RM. Brain metabolism in teenagers with attention-deficit hyperactivity disorder. Arch Gen Psychiatr. 1993 May; 50(5): 333-40.

Ziegler, E, Blocher, D, Groß, I, Rösler, M. Erfassung von Symptomen aus dem Spektrum des Hyperkinetischen Syndroms bei Häftlingen einer Justizvollzugsanstalt. Recht \& Psychiatr. Vol 21(1) 2003, 17-21. 